\title{
Probing mechanism scheduling for connected coverage wireless sensor network.
}

\begin{abstract}
Sensing coverage and network connectivity are two main requirements which maintain perfect operation of wireless sensor network. Joint scheduling method has considered both requirements by using random scheduling for sensing coverage, which divides sensor nodes to $\mathrm{k}$ subsets. Each sensor nodes randomly selects one defined subset. Then, the algorithm turns on extra sensor nodes, if necessary for network connectivity. As Extra-on sensor nodes participate in other nodes routing, some of them may be subject of many times transmission and reception. Furthermore, some of Extra-on nodes should be active the whole time to create network connectivity. Both mentioned reasons can drain out energy of those extra active nodes and may lead to network partitioning. Hence, reducing number of Extra-on nodes is important. In this study, we utilize probing mechanism scheduling in joint scheduling method to reduce the number of extra on sensor nodes. By using probing mechanism that some nodes change their working schedule, number of extra on nodes reduces by $20 \%$
\end{abstract}

Keyword: Wireless sensor networks; Coverage; Scheduling; Partitioning; Connectivity; Probing. 